\title{
CO Emission toward HI Absorption Sources in the Large Magellanic Cloud
}

\author{
M. Marx-Zimmer and F. Zimmer \\ Radioastronomisches Institut der Universität Bonn, Auf dem Hügel 71, \\ 53121 Bonn, Germany \\ U. Herbstmeier \\ Max-Planck-Institut für Astronomie, Königstuhl 17, 69117 Heidelberg, \\ Germany
}

\section{J. M. Dickey}

Department of Astronomy, University of Minnesota, Minneapolis, Minnesota 55455, USA

\section{Introduction}

In the Large Magellanic Cloud (LMC) a large number of cool HI clouds have been detected with temperatures much lower than those found for atomic clouds in the Milky Way (Dickey et al. 1994; Mebold et al. 1997; Marx-Zimmer et al. 1998). Apparently, the population of cool HI clouds reaches kinetic gas temperatures down to as low as 10 or $20 \mathrm{~K}$. These clouds may play an important role in the formation of stars in the LMC. We studied the association between the cool atomic gas and molecular gas in the LMC by ${ }^{12} \mathrm{CO}(1-0)$ line observations in directions of cool HI clouds using the 15-m Swedish-ESO Submillimetre Telescope $\left(\mathrm{SEST}^{1}\right)$.

\section{Observations}

We used the SEST to survey ${ }^{12} \mathrm{CO}(1-0)$ line emission toward 25 lines of sight showing cool $\mathrm{HI}$ ( $57 \mathrm{HI}$ absorption features) with spin temperatures down to $4 \mathrm{~K}$. The sources have been selected from the HI absorption line surveys of Dickey et al. (1994) and Marx-Zimmer et al. (1998). We also studied the surroundings of 13 of these lines of sight by a four-point map around the central position with $40^{\prime \prime}$ spacing. The ${ }^{12} \mathrm{CO}(1-0)$ observations were carried out in a dual beam-switching mode (switching frequency $6 \mathrm{~Hz}$ ) with a beam throw of $11^{\prime} 37^{\prime \prime}$ in azimuth and in a frequency-switching mode with a frequency offset of $15 \mathrm{MHz}\left(40 \mathrm{~km} \mathrm{~s}^{-1}\right)$. At the frequency of $115 \mathrm{GHz}$ of the $\mathrm{J}=1-0$ transition of ${ }^{12} \mathrm{CO}$ the SEST beamwidth is $45^{\prime \prime}$, corresponding to a linear resolution of about $10 \mathrm{pc}$ in the LMC. The velocity resolution is $0.11 \mathrm{~km} \mathrm{~s}^{-1}$. Typical integration times were $20 \mathrm{~min}$ on the source, which provides an rms of about $0.06 \mathrm{~K}$.

\footnotetext{
${ }^{1}$ Based on observations with the Swedish-ESO Submillimetre Telescope (SEST) at the European Southern Observatory (ESO), La Silla, Chile.
} 


\section{Results}

Out of a total sample of 25 lines of sight showing cool atomic hydrogen $\mathrm{CO}$ emission has been detected toward 8 lines of sight with $\mathrm{T}_{M B}$ between $0.3 \mathrm{~K}$ and $8 \mathrm{~K}$. Five of these lines of sight showing $\mathrm{CO}$ emission are in direction of the 30 Doradus complex. The other clouds have been detected toward the bar of the LMC, toward the far southwest, and in direction of LMC4. Four of these clouds have not been detected in the CO survey of Cohen et al. (1988) due to a smaller beam filling factor ( $8^{\prime} .8$ resolution). We mapped five of the detected $\mathrm{CO}$ clouds and found cloud sizes between about $110^{\prime \prime} \times 80^{\prime \prime}(26 \mathrm{pc} \times 19 \mathrm{pc})$ and $600^{\prime \prime} \times 180^{\prime \prime}(144 \mathrm{pc} \times 43 \mathrm{pc})$. The cool HI gas is mostly located toward the rim of the molecular clouds.

There is no clear correlation of $\mathrm{CO}$ emission with the HI spin temperature or the optical depth, $\tau_{H I}$, although the probability of finding a CO-HI association increases with $\tau_{H I}$ and with the absorption integral. CO emission always occurs near but does not necessarily coincide with an $\mathrm{HI}$ absorption feature. The velocity differences $D v=v_{H I}-v_{C O}$, where $v_{H I}$ is the HI feature closest in velocity to the $\mathrm{CO}$ feature, are between 1 and $6 \mathrm{~km} \mathrm{~s}^{-1}$. In general the value is smaller than the FWHM of the HI feature detected. Turbulent gas motion near 30 Doradus and LMC4 can cause this velocity shift of the CO gas with respect to the HI gas.

Although LMC clouds seem to have lower temperatures than atomic clouds in the Milky Way, the fraction of cool HI clouds showing CO emission is the same as in the Milky Way, where about $19 \%$ of the $\mathrm{HI}$ absorption components show CO emission (Despois \& Baudry 1985). Whereas in the Milky Way the transition from atomic to molecular gas $\left(\mathrm{H}_{2}, \mathrm{CO}\right)$, leaving little $\mathrm{HI}$, seems to take place at temperatures below about 30 to $40 \mathrm{~K}$, the non-detection of $\mathrm{CO}$ towards unusually cool $\mathrm{HI}$ clouds in the LMC might indicate that here the gas phase transition is determined by a different temperature-density combination. Another explanation of our result is that the conversion factor from $\mathrm{CO}$ emission to $\mathrm{H}_{2}$ column density is lower in the LMC than in the Milky Way. The unusually cool HI clouds may be the key in understanding the high star formation activity in the LMC.

Acknowledgments. M. Marx-Zimmer thanks the financial support through the DLR (Deutsches Zentrum für Luft- und Raumfahrt e.V.) grant 50OR9615.

\section{References}

Cohen, R.S., Dame, M.T., Garay, G., et al. 1988, ApJ, 331, L95

Despois D., \& Baudry, A. 1985, A\&A, 148, 83

Dickey, J.M., Mebold, U., Marx, M., Amy, S., Haynes, R.F., \& Wilson, W. 1994, A\&A, 289, 357

Marx-Zimmer, M., Herbstmeier, U., Zimmer, F., Dickey, J.M., Staveley-Smith, L., \& Mebold, U. 1998, Proceedings of the Workshop The Magellanic Clouds and other Dwarf Galaxies, p. 275

Mebold, U., Düsterberg, C., Dickey, J.M., Staveley-Smith, L., \& Kalberla, P. 1997, A\&A, 490, 65L 